\title{
Persistence of correlations in many-body localized spin chains
}

\author{
Vasilii Vadimov, ${ }^{1,2,3}$ Tapio Ala-Nissila, ${ }^{2,4}$ and Mikko Möttönen $\oplus^{1,5}$ \\ ${ }^{1}$ QCD Labs, QTF Centre of Excellence, Department of Applied Physics, Aalto University, P.O. Box 13500, \\ FI-00076 Aalto, Espoo, Finland \\ ${ }^{2}$ MSP Group, QTF Centre of Excellence, Department of Applied Physics, Aalto University, P.O. Box 11000, FI-00076 Aalto, Espoo, Finland \\ ${ }^{3}$ Institute for Physics of Microstructures, Russian Academy of Sciences, 603950 Nizhny Novgorod, GSP-105, Russia \\ ${ }^{4}$ Interdisciplinary Centre for Mathematical Modelling, Department of Mathematical Sciences, Loughborough University, \\ Loughborough LE11 3TU, United Kingdom \\ ${ }^{5}$ VTT Technical Research Centre of Finland Ltd., QTF Center of Excellence, P.O. Box 1000, FI-02044 VTT, Finland
}

(Received 14 July 2020; revised 14 September 2020; accepted 5 October 2020; published 29 October 2020)

\begin{abstract}
We study the evolution and persistence of quantum and classical correlations between spatially separated sites in the disordered XXZ spin chain in its ergodic and many-body-localized phases using exact diagonalization and matrix product state simulations. We show that in the many-body-localized phase, quantum entanglement survives in long-time unitary dynamics of the system, even though it is more fragile than the classical correlations. We study the dependence of the residual entanglement and correlations on the system size and on the disorder strength. Finally, we demonstrate the robustness of entanglement and correlations with respect to local dissipation in the bulk of the system.
\end{abstract}

DOI: 10.1103/PhysRevResearch.2.043154

\section{INTRODUCTION}

Quantum correlations [1] that contain and preserve information beyond classical capabilities play an important role in a variety of interacting quantum systems and quantum devices, such as quantum computers [2]. The two flavors of quantum correlations, entanglement, and dissonance, jointly known as quantum discord, can be characterized by measures, such as concurrence, mutual information, and relative entropy between the states [3]. Entanglement in particular is a fragile property: Interactions with the environment inevitable in real systems typically leads to, at least, an exponential decay of entanglement in time. In some cases, entanglement sudden death (ESD) can occur, leading to a complete destruction of entanglement in a finite time [4,5]. Development of a long-term quantum memory calls for the preservation of entanglement for extended times and preventing ESD in particular.

Related to a quantum memory, Ref. [6] studies the dynamics of a single bit of classical or quantum information in a disordered spin chain. In such systems there is a transition from the ergodic phase to a many-body-localized (MBL) phase with the increase in the disorder strength [7-41]. The MBL phase is an extension of the Anderson-localized state to the case of interacting particles in a disordered potential $[42,43]$. In ergodic systems the local observables contain no information about the initial state after long unitary evolution, which is detrimental to the preservation of correlations

Published by the American Physical Society under the terms of the Creative Commons Attribution 4.0 International license. Further distribution of this work must maintain attribution to the author(s) and the published article's title, journal citation, and DOI.
[44-46]. However, strong disorder may prevent equilibration of the system, and, consequently, it may be possible to recover some information on the initial state using only local measurements. This property of the MBL phase of matter, together with the our results on the persistence of entanglement discussed below, render the MBL physics a potential candidate for improving quantum memories.

\section{MODEL}

In this paper, we study the dynamics of classical correlations and entanglement in the XXZ spin chain with on-site disorder. The dynamics of the spin chain is governed by the Hamiltonian,

$$
\hat{H}=\sum_{j=1}^{N} \frac{\hbar \omega_{j}}{2} \hat{\sigma}_{j}^{z}+\frac{\hbar J}{4} \sum_{j=1}^{N-1}\left(\hat{\sigma}_{j}^{x} \hat{\sigma}_{j+1}^{x}+\hat{\sigma}_{j}^{y} \hat{\sigma}_{j+1}^{y}+\Delta \hat{\sigma}_{j}^{z} \hat{\sigma}_{j+1}^{z}\right)
$$

where $\hat{\sigma}_{j}^{\alpha}, \alpha=x, y, z$ are the Pauli spin-1/2 operators corresponding to the site $j, \omega_{j}$ is the single-spin potential at site $j, J$ is referred to as the spin-spin coupling constant and $\Delta$ is the anisotropy of the spin-spin interaction. Here, we mostly focus on uncorrelated random potential (RP) for which $\left\{\omega_{j}\right\}$ are independent random numbers uniformly distributed within the range $[-J \Omega, J \Omega]$. We also consider the case of quasiperiodic potential (QPP) with $\omega_{j}=J \Omega \cos (2 \pi \alpha j+\varphi)$, where $\alpha=\alpha_{\mathrm{qp}}$ is an irrational number and $\varphi$ is a random phase. We choose $\alpha_{\mathrm{qp}}$ to be the lesser golden ratio $(\sqrt{5}-1) / 2$. Furthermore, we study the case of periodic potential (PP) given by the same expression as for the QPP, but $\alpha=\alpha_{p}$ is a rational number. We choose $\alpha_{p}$ to be equal to 0.6 which yields a period of the potential equal to 5 . 
In the case of the RP and isotropic coupling $\Delta=1$, the transition between the ergodic and the MBL phases is expected to take place at $\Omega \approx 3.5[9,13,23,28]$. However, there are some suggestions that this value of the critical disorder strength may be an underestimate [36]. For the XXZ spin chain in the QPP, Refs. [29,38-41] imply the MBL transition to occur roughly at $\Omega \approx 3$ for the anisotropy $\Delta=0.25$. In the case of the, PP there is no MBL phase in the thermodynamic limit owing to conservation of quasimomentum. However, if the localization length $\xi$ is much shorter than the period of the potential $L$, the thermalization time of the full length chain is expected to scale exponentially as $\propto \exp (L / \xi)$. Thus, in properly engineered periodic systems, we may expect similar dynamics as in the quasiperiodic chains.

Dynamics of quantum information in the MBL systems has been previously studied, for example, in Refs. [47-49], and we follow the scenario laid out in the Ref. [6]. We focus here on the dynamics of a pair of spins located at the ends of a linear chain of $N$ spins in total. The rest of the spins at sites $2, \ldots, N-1$ play the role of a non-Markovian and possibly nonergodic bath, effects pronounced at high $\Omega$. We initialize each bath spin into the maximally mixed infinitetemperature state. The initial state of the pair is chosen to be either a mixed classically correlated (CC) state with the reduced density operator $\hat{\rho}_{1 N}^{\mathrm{CC}}=(|\uparrow \downarrow\rangle\langle\uparrow \downarrow|+| \downarrow \uparrow\rangle\langle\downarrow \uparrow|) / 2$ or the Bell state $\hat{\rho}_{1 N}^{\text {Bell }}=(|\uparrow \downarrow\rangle+|\downarrow \uparrow\rangle)(\langle\uparrow \downarrow|+\langle\downarrow \uparrow|) / 2$. These two states have equal single-spin reduced density operators and the results of spin measurements in the $\{|\uparrow\rangle,|\downarrow\rangle\}$ basis are perfectly anticorrelated. However, the important difference between these states is that the Bell state is entangled, i.e., it has quantum correlations, whereas the $\mathrm{CC}$ state carries only classical correlations. The density operators of the whole system corresponding to these initial conditions read as follows:

$$
\begin{gathered}
\hat{\rho}_{0}^{\mathrm{CC}}=\frac{1}{2^{N}}\left(\mathbb{1}-\hat{\sigma}_{1}^{z} \hat{\sigma}_{N}^{z}\right), \\
\hat{\rho}_{0}^{\text {Bell }}=\frac{1}{2^{N}}\left(\mathbb{1}-\hat{\sigma}_{1}^{z} \hat{\sigma}_{N}^{z}+\hat{\sigma}_{1}^{x} \hat{\sigma}_{N}^{x}+\hat{\sigma}_{1}^{y} \hat{\sigma}_{N}^{y}\right),
\end{gathered}
$$

where $\mathbb{1}$ is the $N$-spin identity operator.

The unitary evolution of the density operator is governed by the Liouville-von Neumann equation as

$$
\frac{d \hat{\rho}}{d t}=-\frac{i}{\hbar}[\hat{H}, \hat{\rho}] .
$$

We quantify the correlations between the outermost spins of the chain with mutual information [50] and concurrence [51,52] which are common measures of the total correlations and quantum entanglement between the spins of the pair, respectively. Concurrence is nonzero only if the spins are entangled, whereas mutual information vanishes only if the spins are uncorrelated. Mutual information is defined as $I=$ $S\left(\hat{\rho}_{1}\right)+S\left(\hat{\rho}_{N}\right)-S\left(\hat{\rho}_{1 N}\right)$ where $S(\hat{\rho})=-\operatorname{Tr} \hat{\rho} \ln \hat{\rho}$ is the von Neumann state entropy, and $\hat{\rho}_{1}$ and $\hat{\rho}_{N}$ are the reduced density operators of the first and the last spin, respectively. Concurrence is equal to $C=\max \left(0, \lambda_{1}-\lambda_{2}-\lambda_{3}-\lambda_{4}\right)$, where $\lambda_{j}$ are the eigenvalues of the matrix $R=\sqrt{\sqrt{\hat{\rho}_{1 N}} \hat{\tilde{\rho}}_{1 N} \sqrt{\hat{\rho}_{1 N}}}$ in the descending order and $\hat{\tilde{\rho}}_{1 N}=\left(\hat{\sigma}^{y} \otimes \hat{\sigma}^{y}\right) \hat{\rho}_{1 N}^{*}\left(\hat{\sigma}^{y} \otimes \hat{\sigma}^{y}\right)$ is the time-reversed reduced density operator of the spin pair. We expect that the behavior of these quantities is mostly determined by the interaction of the outermost spins with bath spins in their close proximity. This is because in the case of strong disorder the system is expected to be localized and the propagation of correlations is suppressed, and in the case of weak disorder, each spin thermalizes through local interactions with a mostly incoherent bath.

\section{THEORETICAL ANALYSIS}

To obtain initial analytical understanding of the relevant MBL physics, we analyze the dynamics of the spin chain using a phenomenological description of the MBL phase. References [15,22,35] state that the spin operators $\hat{\sigma}_{j}^{z}$ can be transformed into so-called local integrals of motion $\hat{\tau}_{j}^{z}=$ $\hat{U} \hat{\sigma}_{j}^{z} \hat{U}^{\dagger}$ by a certain quasilocal unitary transformation $\hat{U}$. The Hamiltonian of the system (1) can be written in terms of these integrals of motions $[15,22,35]$ as

$$
\hat{H}=\hbar \sum_{j} K_{j}^{(1)} \hat{\tau}_{j}^{z}+\hbar \sum_{j_{1}<j_{2}} K_{j_{1} j_{2}}^{(2)} \hat{\tau}_{j_{1}}^{z} \hat{\tau}_{j_{2}}^{z}+\cdots,
$$

where the coefficients $K_{j_{1}, j_{2}, \ldots, j_{k}}^{(k)}$ decay exponentially with increasing $k$ and $j_{k}-j_{1}$. Assuming the system to be deep in the MBL phase, we neglect the terms with $k \geqslant 3$. In this limit, we also neglect the distinction between the spin operators and the local integrals of motion, and, hence, express the initial conditions as

$$
\begin{gathered}
\hat{\rho}_{0}^{\mathrm{CC}} \approx \frac{1}{2^{N}}\left(\mathbb{1}-\hat{\tau}_{1}^{z} \hat{\tau}_{N}^{z}\right), \\
\hat{\rho}_{0}^{\mathrm{Bell}} \approx \frac{1}{2^{N}}\left(\mathbb{1}-\hat{\tau}_{1}^{z} \hat{\tau}_{N}^{z}+\hat{\tau}_{1}^{x} \hat{\tau}_{N}^{x}+\hat{\tau}_{1}^{y} \hat{\tau}_{N}^{y}\right),
\end{gathered}
$$

where $\hat{\tau}_{j}^{\alpha}=\hat{U} \hat{\sigma}_{j}^{\alpha} \hat{U}^{\dagger}, \alpha=x, y, z$.

We observe that the above $\mathrm{CC}$ state does not evolve in time since its density operator commutes with $\hat{H}$. This means that the classical correlations are well protected in the MBL phase. The system prepared in the Bell state evolves nontrivially, but fortunately the reduced density operator of the outermost spins can be evaluated analytically as

$$
\hat{\rho}_{1 N}^{\text {Bell }}(t)=\frac{1}{4}\left\{\mathbb{1}-\hat{\tau}_{1}^{z} \hat{\tau}_{N}^{z}+\chi(t)\left[\hat{\tau}_{1}^{x}(t) \hat{\tau}_{N}^{x}(t)+\hat{\tau}_{1}^{y}(t) \hat{\tau}_{N}^{y}(t)\right]\right\},
$$

where

$$
\begin{gathered}
\chi(t)=\prod_{j=2}^{N-1} \cos \left(2 K_{1 j}^{(2)} t\right) \cos \left(2 K_{j N}^{(2)} t\right), \\
\hat{\tau}_{j}^{x}(t)=\cos \left(2 K_{j}^{(1)} t\right) \hat{\tau}_{j}^{x}+\sin \left(2 K_{j}^{(1)} t\right) \hat{\tau}_{j}^{y}, \\
\hat{\tau}_{j}^{y}(t)=-\sin \left(2 K_{j}^{(1)} t\right) \hat{\tau}_{j}^{x}+\cos \left(2 K_{j}^{(1)} t\right) \hat{\tau}_{j}^{y},
\end{gathered}
$$

and we assumed that $K_{1 N}^{(2)}=0$. Here, the concurrence is equal to $|\chi(t)|$ which decays in time. The classical correlations which are described by the diagonal elements of the density operator appear to be more robust than entanglement: The results of measurements in the eigenbasis of $\hat{\tau}_{z}$ are perfectly anticorrelated.

The coefficients $K_{1 j}^{(2)}$ and $K_{j N}^{(2)}$ are exponentially suppressed except for those that describe the nearest-neighbor interactions. The values of $K_{12}^{(2)}$ and $K_{N-1, N}^{(2)}$ are expected to be close to $\hbar J \Delta / 4$, thus, corresponding to the $\hbar J \Delta \hat{\sigma}_{z} \hat{\sigma}_{z} / 4$ term in the 

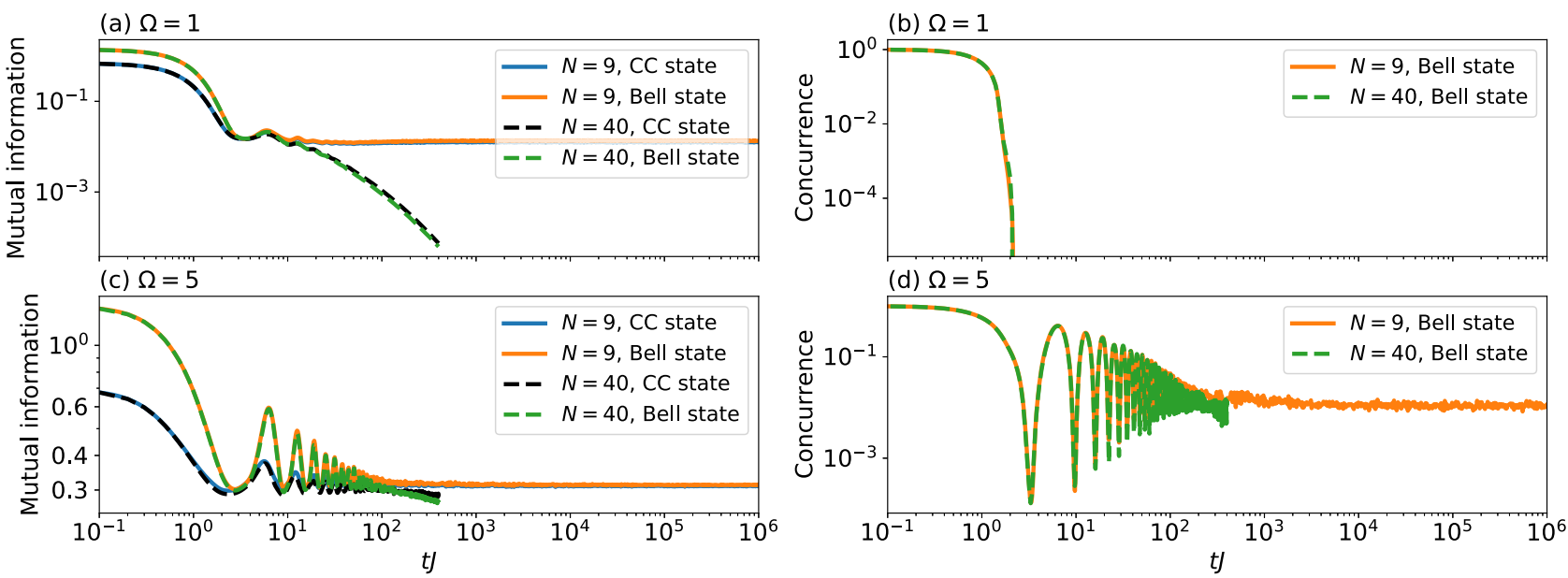

FIG. 1. (a) and (c) Mutual information and (b) and (d) concurrence as functions of time averaged over 400 uncorrelated random realizations of RP with magnitude (a) and (b) $\Omega=1$ and (c) and (d) $\Omega=5$. The anisotropy $\Delta=1$. The data have been obtained using exact diagonalization for $N=9$ and the matrix product states for $N=40$. We show data for both types of initial states, the CC and the Bell states as indicated in the legends.

original Hamiltonian of Eq. (1). In this case, concurrence is proportional to $\cos ^{2}(J \Delta t / 2)$ and, hence, oscillates in time with a period of $2 \pi /(J \Delta)$.

The neglected terms with $k \geqslant 3$ [see Eq. (5)] affect the dynamics of the spin chain at times $t \sim\left(K_{j_{1}, j_{2}, \ldots, j_{k}}^{k}\right)^{-1}$. However, these terms do not affect the persistence of classical correlations because they commute with the density operator of the $\mathrm{CC}$ state. Furthermore, they do not qualitatively change the above-discussed behavior of the entanglement since their main contribution is to cause additional dephasing of the outermost spins at long times. Thus, the concurrence oscillations taking place at short timescales are unaffected by this dephasing.

\section{NUMERICAL RESULTS}

Guided by our above analytical results based on the phenomenological framework we have carried out numerical simulations on the spin chain. We employ the exact diagonalization and matrix product state (MPS) techniques [53-59] in order to simulate the dynamics of short and long spin chains, respectively.

The evolution of mutual information and concurrence between the outermost spins averaged over the RP realizations is shown in Fig. 1. In the case of the only classically correlated initial state, entanglement does not develop during the evolution of the system and concurrence is always zero. For the initially entangled Bell state and high disorder $\Omega=5$, the average concurrence in the MBL phase oscillates with the above-predicted period of $2 \pi /(J \Delta)$ and decays in time. For the longest times considered here, concurrence becomes strongly suppressed but does not completely vanish. This remarkable behavior is in strong contrast to the ESD [4,5] and casts interest on MBL physics from the quantum information point of view.

For the chain with low disorder $\Omega=1$, entanglement between the outermost spins disappears at a finite time indicating ESD. As expected from the phenomenological approach, classical correlations are more robust than the quantum ones and survive in both cases, although at low disorder mutual information at long times is much smaller than at the high disorder. In the case of a long chain $N=40$, we observe the mutual information to monotonously decay in time, but it remains to be studied whether this is an artifact of the MPS method that may underestimate classical and quantum correlations $[6,12]$.

We have further examined the dependence of mutual information and concurrence after a long time of unitary evolution as a function of the chain length. The data are shown in Fig. 2 for $\Omega=5$ in the MBL phase for short and long chains. We observe that the residual mutual information saturates at $N \approx 6$ which appears to be on the order of the localization length at the given disorder strength. In contrast, the analysis utilizing exact diagonalization for the short chains reveals an exponential behavior of the residual concurrence on the chain length whereas the MPS studies show that the concurrence after long evolution is more or less independent on the chain length. This apparent contradiction can be explained by the fact that the evolution times feasible for MPS are much shorter than those available for exact diagonalization. Thus, vanishing of the average concurrence occurs at longer times than we reach in the MPS simulations. Nevertheless, the result that quantum and classical correlations after a long but finite evolution time seem independent on the length of the chain is consistent with the discussion above that only the neighborhood of the outermost spins affects the correlations between them.

In the case of RP, there can be significant realizationdependent local variations in the disorder strength. In particular, if in a random realization there is a region close to an end of the chain where the disorder strength happens to be low, the MBL protection of the outermost spins may be lifted. Consequently, they may thermalize leading to loss of their initial entanglement even though the system as a whole should be in the MBL phase according to the parameter $\Omega$. For QPP defined above, such a situation is much less likely to happen [37]. To study this effect, we compute the fraction of realizations where the entanglement remains nonzero after a long temporal evolution as a function of the $\Omega$, see Fig. 3. For the RP, we find that the crossover between ESD and survival 

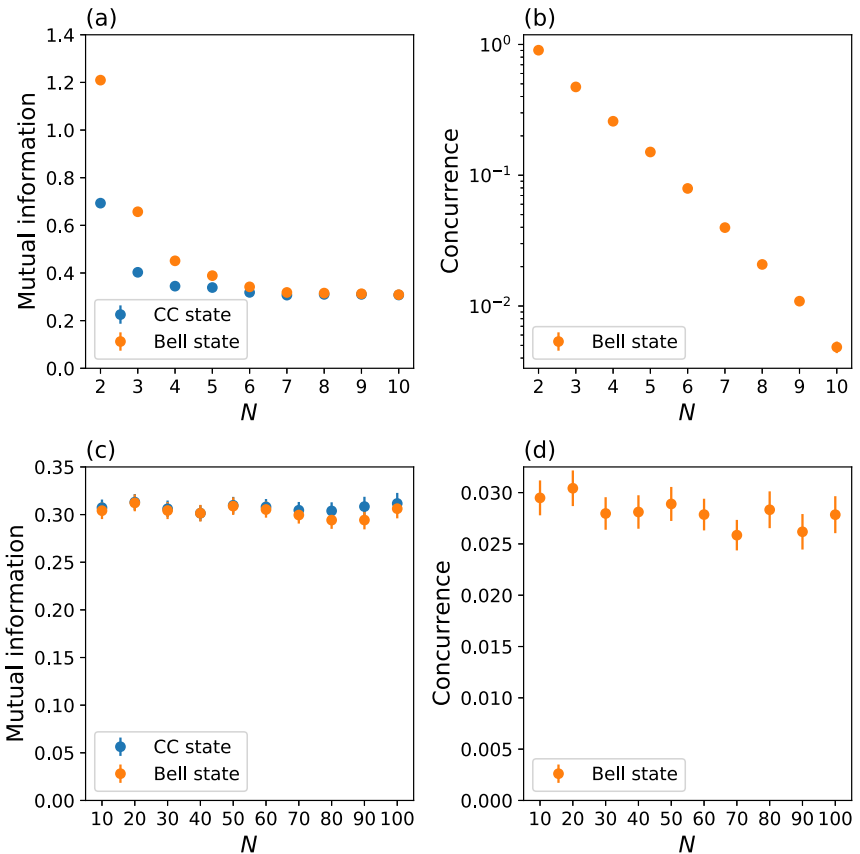

FIG. 2. (a) and (c) Mutual information and (b) and (d) concurrence in the long-time limit as a function of the chain length $N$ in the MBL phase with $\Omega=5$. The data have been averaged over the time interval (a) and (b) $t \in\left[1.3 \times 10^{5}, 10^{6} \mathrm{~J}^{-1}\right]$, (c) and (d) $t \in\left[350\right.$ and $\left.400 \mathrm{~J}^{-1}\right]$ and over $400 \mathrm{RP}$ realizations. The error bars show the error of the mean from the realizations. Here, $\Delta=1$. The total evolution time is (a) and (b) $10^{6} \mathrm{~J}^{-1}$ and (c) and (d) $400 \mathrm{~J}^{-1}$. The dynamics of the chain is simulated using (a) and (b) exact diagonalization or (c) and (d) MPS technique.

of entanglement is broad and there is a significant probability of ESD even at high $\Omega$, well above the supposed MBL transition. For the QPP however, the crossover is sharper and the probability of entanglement survival for given $\Omega$ is much higher than that for the RP. In the case of PP, the probability of entanglement survival behaves in a similar way as for the QPP, especially for the short $N=9$ chains where the curves are identical for the QPP and PP. (a) $N=9$

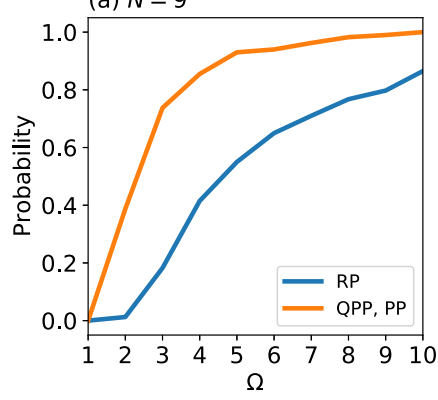

(b) $N=40$

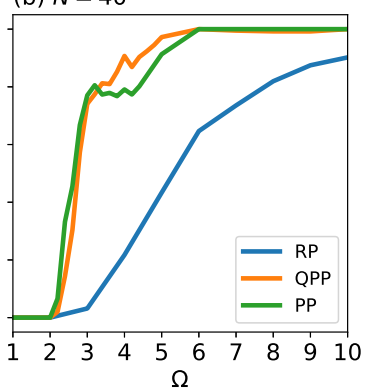

FIG. 3. Probability of entanglement survival as a function of the potential strength $\Omega$ for RP, QPP, and PP as indicated and for (a) a short $N=9$ and (b) a long $N=40$ chain. We assume that the entanglement has survived if there is, at least, one occurrence of nonzero concurrence within the time interval (a) $t \in\left[1.3 \times 10^{5}\right.$ and $\left.10^{6} \mathrm{~J}^{-1}\right]$ or (b) $t \in\left[350\right.$ and $\left.400 \mathrm{~J}^{-1}\right]$. Here, $\Delta=1$ and the data have been gathered for 400 potential realizations.
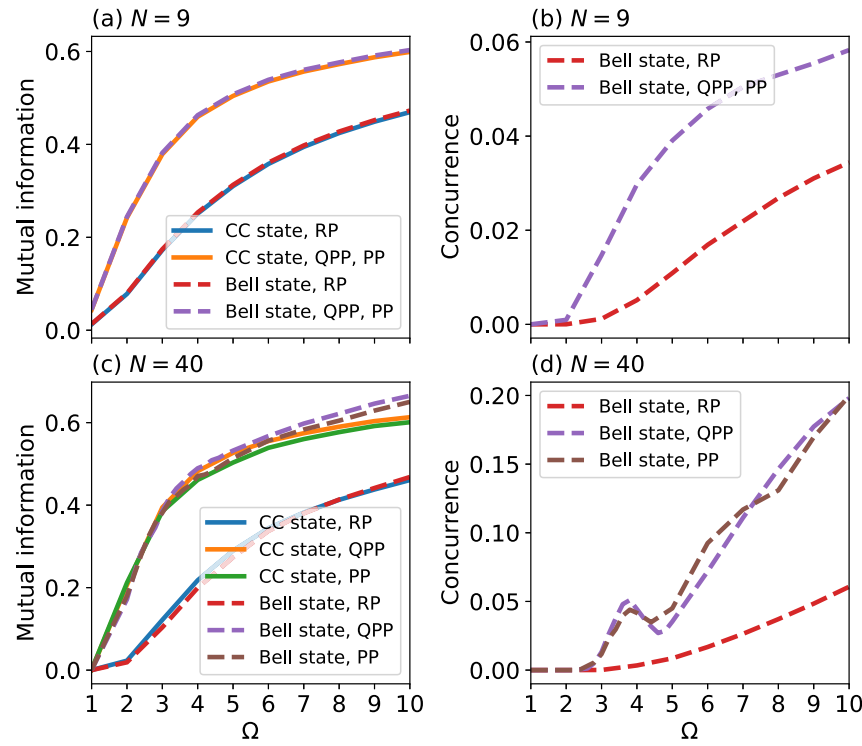

FIG. 4. (a)-(c) Mutual information and (b)-(d) concurrence after long evolution as functions of the potential strength $\Omega$ for a short $N=9$ chain in (a) and (b) and for a long $N=40$ chain in (c) and (d). The data have been averaged over 400 potential realizations and over the time interval (a) and (b) $t \in\left[1.3 \times 10^{5}\right.$ and $\left.10^{6} \mathrm{~J}^{-1}\right]$ and (c) and (d) $t \in\left[350\right.$ and $\left.400 \mathrm{~J}^{-1}\right]$. Here, $\Delta=1$.

In Fig. 4, we study concurrence and mutual information after long unitary evolution as a function of the potential strength $\Omega$. We observe that for the RP both the residual mutual information and concurrence increase monotonically with $\Omega$. The nonmonotonic behavior of concurrence for the long chain $(N=40)$ in the QPP and PP is most likely an artifact of the relatively short evolution time since there is no such feature in the case of the short $(N=9)$ chain for which the evolution may be computed for arbitrary long time thanks to exact diagonalization. Note that the residual mutual information between the outermost spins is almost equal for the initial CC and Bell states. We attribute this coincidence to the fact that both initial states include an equal amount of classical correlations and that the quantum correlations, property of the Bell state only become strongly suppressed during the temporal evolution while classical correlations persist.

Interestingly, Figs. 3 and 4 indicate that the residual entanglement and the probability of entanglement survival deviate from zero at disorder strengths clearly below the anticipated transition point $\Omega_{*} \approx 3.5$ for the RP. In the case of short chains, this is likely to be a finite-size effect. For the long chains, the reason of this effect may be that the thermalization time becomes much longer than our simulations times when approaching the transition from the ergodic side [27].

\section{EFFECT OF AN ADDITIONAL MARKOVIAN BATH}

In physical systems, there are usually multiple decay channels, such as coupling to a thermal bath of electrons and/or phonons causing dephasing of the quantum states and affecting localization [60,61]. To study this effect, we choose here that a spin labeled by the index $j_{\mathrm{d}}$ is connected to a Markovian bath of infinite temperature. In the limit of weak 


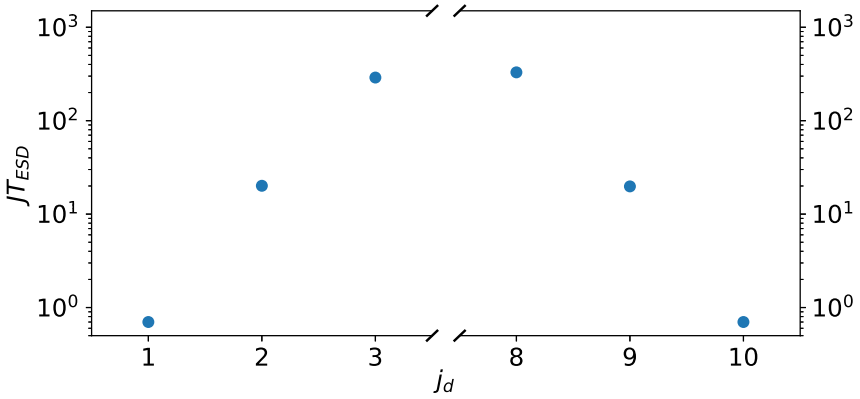

FIG. 5. Time of ESD as a function of the position of the dissipating spin $j_{\mathrm{d}}$. Here, $N=10, \Omega=5, \Delta=1$, and $\gamma=J / 2$. The calculation time is $10^{3} \times J^{-1}$. In the cut region where $3<j_{d}<8$, the time of ESD exceeds the longest time period considered.

spin-bath coupling, such a system can be approximated by the well-known Lindblad equation,

$$
\frac{d \hat{\rho}}{d t}=-\frac{i}{\hbar}[\hat{H}, \hat{\rho}]+2 \gamma\left(\hat{\sigma}_{j_{d}}^{-} \hat{\rho} \hat{\sigma}_{j_{d}}^{+}+\hat{\sigma}_{j_{d}}^{+} \hat{\rho} \hat{\sigma}_{j_{d}}^{-}-\hat{\rho}\right),
$$

where $\hat{\sigma}_{j}^{ \pm}=\left(\hat{\sigma}_{j}^{x} \pm i \hat{\sigma}_{j}^{y}\right) / 2$ and $\gamma$ is the coupling strength. We solve the Lindblad equation numerically for the $\mathrm{XXZ}$ chain and study the dependence of the time of ESD on the position of the dissipating spin $j_{\mathrm{d}}$, see Fig. 5. In the localized phase with $\Omega=5$ and $\gamma=J / 2$, if the dissipating spin is a spin of the initially mutually correlated pair, the entanglement rapidly dies after time comparable to $1 / \gamma$. However, if we move the position of the dissipative spin towards the bulk of the chain, the time it takes to observe ESD seems to increase exponentially and becomes inaccessible for the computationally feasible evolution times of the MPS scheme. This study suggests that potential coupling of the bulk spins to their Markovian baths does not change our above results on the persistence of entanglement between the outermost spins.

\section{SUMMARY AND CONCLUSIONS}

We have studied the dynamics of entanglement and classical correlations of a pair of spins located at the ends of a linear XXZ spin chain with disorder. We have quantified the amount of quantum and classical correlations between this pair with concurrence and mutual information. Remarkably, both of these quantities survive in the strongly disordered MBL phase, even in the long-time limit. We have also quantified the dependence of residual concurrence and mutual information after long temporal evolution on the chain length and the disorder strength. We have shown that the residual correlations in the MBL phase do not depend on the bath size, which confirms the intuitive picture that the dynamics of the outermost spins is determined by their close-by environment. We have also shown that residual quantum and classical correlations increase with the disorder strength, but there is a significant difference in the case of uncorrelated random and quasiperiodic potential. Finally, we have demonstrated that provided the chain is in the MBL phase, the correlations between the outermost spins are protected from local dissipation in the bulk of the chain. These observations suggest that understanding MBL physics is potentially useful in the future endeavor of building large-scale quantum processors and especially memories where distant qubits may be entangled. In fact, we have verified that the observed protection of the entanglement is not limited to random or quasiperiodic potential, but quantitatively very similar results may be obtained in a periodic structure provided that the qubit frequencies are chosen correctly. In the future, we plan to extend our calculations to typical qubits, such as transmons taking into account their technical details.

\section{ACKNOWLEDGMENTS}

We thank I. M. Khaymovich for fruitful discussions. This research was financially supported by the European Research Council under Grant No. 681311 (QUESS) and by the Academy of Finland through its Centre of Excellence in Quantum Technology (QTF) (Grants No. 312298 and No. 312300). We acknowledge the computational resources provided by the Aalto Science-IT project, CSC-IT Center for Science, Finland, and Max Planck Institute for Physics of the Complex Systems, Dresden, Germany. The template-based Armadillo library [62] was utilized in the implementation of the MPS algorithm.

\section{APPENDIX: COMPUTATIONAL METHODS INVOLVING MATRIX PRODUCT STATES}

We describe the density operator of the chain using the MPS representation [53-59] of the density operator as

$$
\begin{aligned}
\hat{\rho}= & \sum_{\alpha_{k}=1}^{D} \sum_{s_{j} \in\{0, x, y, z\}} A_{\alpha_{1}}^{s_{1}} A_{\alpha_{1} \alpha_{2}}^{s_{2}} \ldots A_{\alpha_{N-1}}^{s_{N}} \\
& \times \frac{\hat{\sigma}_{1}^{s_{1}} \hat{\sigma}_{2}^{s_{2}} \cdots \hat{\sigma}_{N}^{s_{N}}}{\sqrt{2^{N}}},
\end{aligned}
$$

where the dimension of the $D \times D$ matrices $A_{\alpha_{j-1} \alpha_{j}}^{s_{j}}$ is referred to as the bond dimension $D$ of the MPS representation and the first $A_{\alpha_{1}}^{s_{1}}$ and the last $A_{\alpha_{N-1}}^{s_{N}}$ matrices are row and column vectors, respectively, so that the product in Eq. (A1) is a scalar. The accuracy of the MPS ansatz increases with the bond dimension. Since the density operator is Hermitian all the matrices in the representation are real.

The density operator obeys the Liouville-von Neumann or Lindblad equations for the closed or open system, respectively. These equations can be formally written as

$$
\frac{d \hat{\rho}}{d t}=\mathcal{L} \hat{\rho},
$$

where the Liouvillian $\mathcal{L}$ can be expressed as a matrix product operator (MPO) in the following form:

$$
\begin{aligned}
\mathcal{L} \hat{\rho}= & \frac{1}{2^{N}} \sum_{\alpha_{k}=1}^{D} \sum_{s_{j}, s_{k}^{\prime} \in\{0, x, y, z\}} W_{\alpha_{1}}^{s_{1} s_{1}^{\prime}} W_{\alpha_{1} \alpha_{2}}^{s_{2} s_{2}^{\prime}} \cdots W_{\alpha_{N-1}}^{s_{N} s_{N}^{\prime}} \\
& \times \hat{\sigma}_{1}^{s_{1}} \hat{\sigma}_{2}^{s_{2}} \cdots \hat{\sigma}_{N}^{s_{N}} \operatorname{Tr}\left(\hat{\sigma}_{1}^{s_{1}^{\prime}} \hat{\sigma}_{2}^{s_{2}^{\prime}} \cdots \hat{\sigma}_{N}^{s_{N}^{\prime}} \hat{\rho}\right),
\end{aligned}
$$

where $W_{\alpha_{j-1} \alpha_{j}}^{s_{j} s_{k}^{\prime}}, W_{\alpha_{1}}^{s_{1} s_{1}^{\prime}}$, and $W_{\alpha_{N-1}}^{s_{N} s_{N}^{\prime}}$ are real $D \times D, 1 \times D$, and $D \times 1$ coefficient matrices, respectively. Since the Hamiltonian of Eq. (1) has only short-range interactions, the corresponding Liouvillian can be exactly expressed in the MPO form with $O(1)$ bond dimension. 
To integrate Eq. (A2) over a time interval $[t, t+\delta t]$, we apply the operator exponential of the Liouvillian, $\exp (\mathcal{L} \delta t)$ to the current state $\hat{\rho}(t)$. For the Hamiltonians with the nearest-neighbor interaction, the evaluation of the operator exponential can be implemented by splitting the Liouvillian into a sum of two terms as

$$
\mathcal{L}=\mathcal{L}_{\mathrm{o}}+\mathcal{L}_{\mathrm{e}}
$$

where

$$
\begin{gathered}
\mathcal{L}_{\mathrm{o} / \mathrm{e}} \hat{\rho}=-\frac{i}{\hbar}\left[\hat{H}_{\mathrm{o} / \mathrm{e}}, \hat{\rho}\right], \\
\hat{H}_{\mathrm{o}}=\frac{\hbar \omega_{1}}{4} \sigma_{1}^{z}+\sum_{j=1}^{\lfloor N / 2\rfloor}\left[\frac{\hbar \omega_{2 j-1}}{4} \hat{\sigma}_{2 j-1}^{z} \frac{\hbar \omega_{2 j}}{4} \hat{\sigma}_{2 j}^{z}\right. \\
\left.+\frac{\hbar J}{4}\left(\hat{\sigma}_{2 j-1}^{x} \hat{\sigma}_{2 j}^{x}+\hat{\sigma}_{2 j-1}^{y} \hat{\sigma}_{2 j}^{y}+\Delta \hat{\sigma}_{2 j-1}^{z} \hat{\sigma}_{2 j}^{z}\right)\right],
\end{gathered}
$$

and

$$
\begin{aligned}
\hat{H}_{\mathrm{e}}= & \sum_{j=1}^{\lfloor(N-1) / 2\rfloor}\left[\frac{\hbar \omega_{2 j}}{4} \hat{\sigma}_{2 j}^{z}+\frac{\hbar \omega_{2 j+1}}{4} \hat{\sigma}_{2 j+1}^{z}\right. \\
& \left.+\frac{\hbar J}{4}\left(\hat{\sigma}_{2 j}^{x} \hat{\sigma}_{2 j+1}^{x}+\hat{\sigma}_{2 j}^{y} \hat{\sigma}_{2 j+1}^{y}+\Delta \hat{\sigma}_{2 j}^{z} \hat{\sigma}_{2 j+1}^{z}\right)\right]+\frac{\hbar \omega_{N}}{4} \sigma_{N}^{z}
\end{aligned}
$$

In our case, all the terms in the above sum for either $\mathcal{L}_{\mathrm{o}}$ or $\mathcal{L}_{\mathrm{e}}$ commute pairwise, and hence, the $\operatorname{exponential} \exp \left(\mathcal{L}_{\mathrm{o} / \mathrm{e}} \delta t\right)$ is a product of the individually exponentiated terms. The result can be efficiently represented as a MPO. Subsequently, we employ the Trotter-Suzuki decomposition for the operator exponential as

$$
e^{\mathcal{L} \delta t}=e^{(1 / 2) \mathcal{L}_{0} \delta t} e^{\mathcal{L}_{\mathrm{e}} \delta t} e^{(1 / 2) \mathcal{L}_{0} \delta t}+O\left(\delta t^{3}\right),
$$

where we omit the term $O\left(\delta t^{3}\right)$ in our calculations. The exact application of the operator exponential to the state $\hat{\rho}$ leads to a rapid exponential growth of the bond dimension in the corresponding MPS representation. Consequently, we compress to the state after each time step into a representation with a fixed maximum bond length.

The MPS approximation works well if the temporally evolving state of the system exhibits so-called area-law entanglement, i.e., the entanglement entropy across some bipartition scales as the area of this biparition, which for the studied one-dimensional systems is just a single point since the maximal achievable entanglement entropy in the MPS representation scales as $\ln D$ [59]. In MBL systems, the entanglement of the eigenstates obeys the area law and the temporal evolution of an initially nonentangled or weakly entangled state shows logarithmic growth of entanglement [9]. This is in stark contrast to ergodic systems which usually support volume-law entangled eigenstates for which the entanglement entropy across a certain biparition is proportional to the volume of the bipartition. These systems also exhibit linear entanglement growth in time [9]. Consequently, the MPS representation with a reasonably small $D$ is accurate for systems in the MBL phase but fails to describe ergodic quantum systems. In fact, the ability to accurately describe the system using MPS representation with low bond dimension may, in some cases, be a signature of the MBL phase [6]. However, at very short times of the evolution of the system, the MPS representation may be accurate enough even for the systems deeply in the ergodic phase since the entanglement does not appear instantly in the system. Since the accuracy of the MPS representation strongly depends on the whether we are in the MBL phase or not, we use different bond dimensions for different disorder strength varying from $D=40$ for $\Omega=$ 10 to $D=100$ for $\Omega=1$. For a representative collection of parameter values, we have numerically verified that within the resulting uncertainty, this technique yields mutual information and concurrence insensitive to further increments of the bond dimension.
[1] F. J. Duarte, Fundamentals of Quantum Entanglement (IOP, Bristol, U.K., 2019), pp. 2053-2563.

[2] F. Arute, K. Arya, R. Babbush, D. Bacon, J. C. Bardin, R. Barends, R. Biswas, S. Boixo, F. G. S. L. Brandao, D. A. Buell, B. Burkett, Y. Chen, Z. Chen, B. Chiaro, R. Collins, W. Courtney, A. Dunsworth, E. Farhi, B. Foxen, A. Fowler, C. Gidney, M. Giustina, R. Graff, K. Guerin, S. Habegger, M. P. Harrigan, M. J. Hartmann, A. Ho, M. Hoffmann, T. Huang, T. S. Humble, S. V. Isakov, E. Jeffrey, Z. Jiang, D. Kafri, K. Kechedzhi, J. Kelly, P. V. Klimov, S. Knysh, A. Korotkov, F. Kostritsa, D. Landhuis, M. Lindmark, E. Lucero, D. Lyakh, S. Mandrà, J. R. McClean, M. McEwen, A. Megrant, X. Mi, K. Michielsen, M. Mohseni, J. Mutus, O. Naaman, M. Neeley, C. Neill, M. Y. Niu, E. Ostby, A. Petukhov, J. C. Platt, C. Quintana, E. G. Rieffel, P. Roushan, N. C. Rubin, D. Sank, K. J. Satzinger, V. Smelyanskiy, K. J. Sung, M. D. Trevithick, A. Vainsencher, B. Villalonga, T. White, Z. J. Yao, P. Yeh, A. Zalcman, H. Neven, and J. M. Martinis, Nature (London) 574, 505 (2019).
[3] K. Modi, T. Paterek, W. Son, V. Vedral, and M. Williamson, Phys. Rev. Lett. 104, 080501 (2010).

[4] T. Yu and J. H. Eberly, Phys. Rev. Lett. 97, 140403 (2006).

[5] T. Yu and J. H. Eberly, Science 323, 598 (2009).

[6] M. C. Bañuls, N. Y. Yao, S. Choi, M. D. Lukin, and J. I. Cirac, Phys. Rev. B 96, 174201 (2017).

[7] I. V. Gornyi, A. D. Mirlin, and D. G. Polyakov, Phys. Rev. Lett. 95, 206603 (2005).

[8] D. M. Basko, I. L. Aleiner, and B. L. Altshuler, Ann. Phys. 321, 1126 (2006).

[9] D. A. Abanin, E. Altman, I. Bloch, and M. Serbyn, Rev. Mod. Phys. 91, 021001 (2019).

[10] V. Oganesyan and D. A. Huse, Phys. Rev. B 75, 155111 (2007).

[11] L. Fleishman and P. W. Anderson, Phys. Rev. B 21, 2366 (1980).

[12] M. Žnidarič, T. Prosen, and P. Prelovšek, Phys. Rev. B 77, 064426 (2008).

[13] A. Pal and D. A. Huse, Phys. Rev. B 82, 174411 (2010). 
[14] J. H. Bardarson, F. Pollmann, and J. E. Moore, Phys. Rev. Lett. 109, 017202 (2012).

[15] M. Serbyn, Z. Papić, and D. A. Abanin, Phys. Rev. Lett. 111, 127201 (2013).

[16] M. Serbyn, Z. Papić, and D. A. Abanin, Phys. Rev. Lett. 110, 260601 (2013).

[17] M. Serbyn, M. Knap, S. Gopalakrishnan, Z. Papić, N. Y. Yao, C. R. Laumann, D. A. Abanin, M. D. Lukin, and E. A. Demler, Phys. Rev. Lett. 113, 147204 (2014).

[18] M. Serbyn, Z. Papić, and D. A. Abanin, Phys. Rev. B 90, 174302 (2014).

[19] R. Vosk and E. Altman, Phys. Rev. Lett. 112, 217204 (2014).

[20] J. A. Kjäll, J. H. Bardarson, and F. Pollmann, Phys. Rev. Lett. 113, 107204 (2014).

[21] D. Pekker, G. Refael, E. Altman, E. Demler, and V. Oganesyan, Phys. Rev. X 4, 011052 (2014).

[22] D. A. Huse, R. Nandkishore, and V. Oganesyan, Phys. Rev. B 90, 174202 (2014).

[23] D. J. Luitz, N. Laflorencie, and F. Alet, Phys. Rev. B 91, 081103(R) (2015).

[24] R. Vasseur, S. A. Parameswaran, and J. E. Moore, Phys. Rev. B 91, 140202(R) (2015).

[25] R. Nandkishore and D. A. Huse, Annu. Rev. Condens. Matter Phys. 6, 15 (2015).

[26] R. Vosk, D. A. Huse, and E. Altman, Phys. Rev, X 5, 031032 (2015).

[27] K. Agarwal, S. Gopalakrishnan, M. Knap, M. Müller, and E. Demler, Phys. Rev. Lett. 114, 160401 (2015).

[28] T. Devakul and R. R. P. Singh, Phys. Rev. Lett. 115, 187201 (2015).

[29] M. Schreiber, S. S. Hodgman, P. Bordia, H. P. Lüschen, M. H. Fischer, R. Vosk, E. Altman, U. Schneider, and I. Bloch, Science 349, 842 (2015).

[30] J. Z. Imbrie, Phys. Rev. Lett. 117, 027201 (2016).

[31] R. Vasseur and J. E. Moore, J. Stat. Mech.: Theory Exp. (2016) 064010.

[32] S. S. Kondov, W. R. McGehee, W. Xu, and B. DeMarco, Phys. Rev. Lett. 114, 083002 (2015).

[33] J.-y. Choi, S. Hild, J. Zeiher, P. Schauß, A. Rubio-Abadal, T. Yefsah, V. Khemani, D. A. Huse, I. Bloch, and C. Gross, Science 352, 1547 (2016).

[34] J. Smith, A. Lee, P. Richerme, B. Neyenhuis, P. W. Hess, P. Hauke, M. Heyl, D. A. Huse, and C. Monroe, Nat. Phys. 12, 907 (2016).

[35] V. Ros, M. Müller, and A. Scardicchio, Nucl. Phys. B 891, 420 (2015).
[36] E. V. H. Doggen, F. Schindler, K. S. Tikhonov, A. D. Mirlin, T. Neupert, D. G. Polyakov, and I. V. Gornyi, Phys. Rev. B 98, 174202 (2018).

[37] S. Gopalakrishnan, K. Agarwal, E. A. Demler, D. A. Huse, and M. Knap, Phys. Rev. B 93, 134206 (2016).

[38] S.-X. Zhang and H. Yao, Phys. Rev. Lett. 121, 206601 (2018).

[39] F. Setiawan, D.-L. Deng, and J. H. Pixley, Phys. Rev. B 96, 104205 (2017).

[40] S. Iyer, V. Oganesyan, G. Refael, and D. A. Huse, Phys. Rev. B 87, 134202 (2013).

[41] M. Lee, T. R. Look, S. P. Lim, and D. N. Sheng, Phys. Rev. B 96, 075146 (2017).

[42] P. W. Anderson, Phys. Rev. 109, 1492 (1958).

[43] E. Abrahams, 50 Years of Anderson Localization (World Scientific, Singapore, 2010).

[44] J. M. Deutsch, Phys. Rev. A 43, 2046 (1991).

[45] M. Srednicki, Phys. Rev. E 50, 888 (1994).

[46] H. Kim and D. A. Huse, Phys. Rev. Lett. 111, 127205 (2013).

[47] F. Iemini, A. Russomanno, D. Rossini, A. Scardicchio, and R. Fazio, Phys. Rev. B 94, 214206 (2016).

[48] S. Campbell, M. J. Power, and G. De Chiara, European Phys. J. D 71, 206 (2017).

[49] G. De Tomasi, S. Bera, J. H. Bardarson, and F. Pollmann, Phys. Rev. Lett. 118, 016804 (2017).

[50] S. Alipour, S. Tuohino, A. T. Rezakhani, and T. Ala-Nissila, Phys. Rev. A 101, 042311 (2020).

[51] S. Hill and W. K. Wootters, Phys. Rev. Lett. 78, 5022 (1997).

[52] W. K. Wootters, Phys. Rev. Lett. 80, 2245 (1998).

[53] F. Verstraete, J. J. Garcia-Ripoll, and J. I. Cirac, Phys. Rev. Lett. 93, 207204 (2004).

[54] M. Zwolak and G. Vidal, Phys. Rev. Lett. 93, 207205 (2004).

[55] B. Pirvu, V. Murg, J. I. Cirac, and F. Verstraete, New J. Phys. 12, 025012 (2010).

[56] F. Pollmann, V. Khemani, J. I. Cirac, and S. L. Sondhi, Phys. Rev. B 94, 041116(R) (2016).

[57] T. Prosen and M. Žnidarič, J. Stat. Mech.: Theory Exp. (2009) P02035.

[58] M. Žnidarič, A. Scardicchio, and V. K. Varma, Phys. Rev. Lett. 117, 040601 (2016).

[59] U. Schollwöck, Ann. Phys. 326, 96 (2011).

[60] A. Rubio-Abadal, J.-y. Choi, J. Zeiher, S. Hollerith, J. Rui, I. Bloch, and C. Gross, Phys. Rev. X 9, 041014 (2019).

[61] R. Nandkishore and S. Gopalakrishnan, Ann. Phys. 529, 1600181 (2017).

[62] C. Sanderson and R. Curtin, J. Open Source Software 1, 26 (2016). 